\title{
Hydrogen production by the Westinghouse cycle: modelling and optimization of the two-phase electrolysis cell
}

\author{
S. Charton ${ }^{1}$, P. Rivalier ${ }^{1}$, D. Ode ${ }^{1}$, J. Morandini ${ }^{2} \&$ J. P. Caire ${ }^{3}$ \\ ${ }^{I}$ Commissariat à l'Énergie Atomique, Marcoule - DTEG/SGCS/LGCI, \\ France \\ ${ }^{2}$ Astek Rhône-Alpes, France \\ ${ }^{3}$ LEPMI, Grenoble INP, France
}

\begin{abstract}
Hydrogen is currently viewed as a promising energy carrier for transportation applications. In this context, mass production of hydrogen is a major issue for the coming decades. Among the viable production processes, the hybrid sulphur cycle, or Westinghouse cycle, is studied by the Nuclear Energy Division of the French CEA. In this work, the influence of $\mathrm{H}_{2}$ bubbles on the current distribution within the electrolyser is studied. Turbulent two-phase flow simulations are performed with Ansys-Fluent CFD code in the 3D calculation domain using an Euler-Euler model. The electrokinetic problem is solved in the same domain by a finite element code, Flux-Expert, which is able to compute the secondary current distribution by means of specific interfacial elements. Parameters including the cell orientation (vertical or horizontal electrode), flow regime and bubble size are investigated, and the current model development status and needs are discussed.
\end{abstract} Keywords: two-phase flow, numerical model, hydrogen, fluent, flux-expert.

\section{General context}

Hydrogen is a unique zero carbon content energy vector. Its conversion into power and electricity within fuel cells would allow various applications, especially for transportation. Together with fuel cell development and hydrogen storage, hydrogen production is a major issue for the coming decades. The obvious inexhaustible feedstock for hydrogen production is water. Among the 
potential thermochemical cycles able to split water into hydrogen and oxygen, the Westinghouse process appears particularly attractive for its low theoretical energy requirement (in association with a solar or nuclear high temperature source) and its minimal reagent inventory.

The hybrid sulphur cycle (HyS) was developed and patented in the 1970s by the Westinghouse Electric Corporation and, since 2000, has been the subject of renewed interest, especially in the USA and in France. It consists of two steps: an electrochemical step based on sulphur dioxide $\left(\mathrm{SO}_{2}\right)$ electro-oxidation into $\mathrm{H}_{2} \mathrm{SO}_{4}\left(\mathrm{E} 0=0,17 \mathrm{~V}_{\mathrm{SHE}}\right.$ under normal conditions), accompanied by hydrogen gas $\left(\mathrm{H}_{2}\right)$ production at the cathode, and a thermochemical step in which sulphuric acid is decomposed at high temperature to recover $\mathrm{SO}_{2}$, which is recycled to the electrolyser. Among the phenomena and parameters that must be controlled and optimized in order to meet the requirements of hydrogen mass production, this paper focuses on the issues and modelling of two-phase forced convection flow through the electrochemical cell.

\section{Overview of electrolyser modelling approaches}

Numerical models for electrochemical process performance assessment or dimensioning generally assume uniform properties or one-dimensional property variations. For example, plug flow with axial dispersion is usually assumed within filter-press electrolysers [1], whereas a Darcy flow model is commonly used within the gas diffusion layer of PEM electrolysers and fuel cells [2].

Industrial electrochemical cells, for which the local current distribution must be precisely determined, mainly involve multidimensional approaches. The electrokinetic problem is then solved using specific finite element codes such as $\mathrm{COMSOL}^{\mathrm{TM}}$ or Flux-Expert ${ }^{\mathrm{TM}}$.

Conversely, when hydrodynamic phenomena are predominant or limiting with regard to electrochemical reactions, the flow field is modelled by CDF and the electrokinetic problem is simplified.

Focusing on gas-producing electrolysers, Mandin et al. [3] have adopted a Lagrangian approach using Fluent ${ }^{\mathrm{TM}}$ while Agranat et al. [4] have chosen a true two-phase approach using the Euler-Euler algorithm available in PHOENICS ${ }^{\mathrm{TM}}$. The same model was also implemented by Mat et al. [5], who solved the ionic species transport as well in order to derive the tertiary current density distribution within the simulation domain. In ref. [3] the electrical potential is calculated as a user-defined scalar undergoing a diffusion-like transport equation using Dirichlet boundary conditions at electrodes to obtain a primary current distribution. Chemical species transport is modelled neither in ref. [4], where the current density is assumed to be constant at the electrode surfaces, nor in ref. [5]. The simulation domain is generally two-dimensional and often reduced to single fluid zone. Bubbles are assumed to behave as rigid spheres of constant and uniform size.

A few models combine a refined description of both the electrokinetic and hydrodynamic processes. Multiphysics problems are solved by coupling specific codes, either indirectly [6] or by enabling data exchange during the iterative 
process by means of user-defined library routines. The latter formalism was employed by Jomard et al. [7] who coupled Flux-Expert ${ }^{\mathrm{TM}}$ and Fluent ${ }^{\mathrm{TM}}$ to account for the effect of $\mathrm{H}_{2}$ bubble release on the overall performance of the Westinghouse electrolysis process. The same methodology is employed here.

Although gas-evolving electrodes are frequently used in industrial processes, the behaviour of electrogenerated bubbles remains difficult to predict and model. In industrial reactors, large gas release may however strongly influence the electrolyser performance due to hydrodynamic modifications [8] and decreases electrolyte conductivity. These phenomena are examined here, where the process sensitivity to either operating conditions or unknown parameters, such as $\mathrm{H}_{2}$ bubble size and departure angle, is investigated.

More fundamental aspects, such as bubble nucleation, growth and departure, are currently being studied within the scope of the AMELHYFLAM (AMELHYFLAM is the French acronym for Hydrogen, Fluorine and Alumina industrial production processes improvement by coupled modelling of biphasic and electrochemical phenomena) project, supported by the National Research Agency (ANR) and dedicated to this theme.

\section{Model description}

Although the Fluent ${ }^{\mathrm{TM}}$ user-defined functions have been modified in order to optimize data collection and source allocation in the two-phase domain, both the modelling strategy and the simulation domain are similar to the ones developed by Jomard et al. [7]. Hence, only the relevant information and new results are reported here. The exchange data formalism between the two codes is therefore not described.

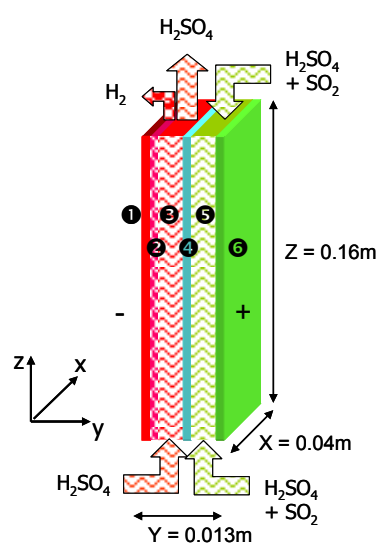

Figure 1: Filter-press electrolyser configuration (1) cathode, 2 hydrogen release zone, $\mathbf{3}$ catholyte, $\mathbf{4}$ membrane, $\mathbf{5}$ anolyte, $\mathbf{6}$ anode). 


\subsection{Description of the electrochemical cell}

The three-dimensional simulation domain is described Figure 1 for a twocompartment filter-press electrolyser. The cathode and anode compartments are separated by a rigid impermeable membrane, to prevent $\mathrm{SO}_{2}$ crossover. The conductivity of a CMX Neocepta ${ }^{\circledR}$ membrane is assumed for simulation. Both $\mathrm{Pt}$ electrodes are flat and parallel. Counter-current fluid flow is maintained under steady-state conditions in the plane-parallel compartments. The dimensions are those of the FM01-LC model, manufactured by ICI Chemical \& Polymer Company, and used in the Westinghouse pilot test facility implemented in the Marcoule Laboratory [9].

\subsection{Simplifying assumptions}

Fluid flow and local mass transport effects in the FM01-LC cell has been investigated by Brown et al. [10] in the case of cupric ions reduction to copper using copper-printed segmented electrodes in the longitudinal or transverse directions. Experiments were carried out under laminar inflow conditions, in the range $212<\operatorname{Re}<855$, in a single compartment cell. According to their experimental results, the authors concluded that it is essential to use a turbulent promoter in order to avoid large variations in the current distribution both along and across the flow direction, due to insufficient radial mixing and to the inlet distributor pattern. Moreover, tracer experiments performed by Trinidad et al. [1] in the range $900<\mathrm{Re}<1900$ exhibits plug flow with axial dispersion behaviour and no evidence of dead zone or fluid channelling when a turbulent promoter is used.

In the process under study here, where $\mathrm{SO}_{2}$ oxidation is the limiting half reaction, the electrolyte flow rates are controlled by volumetric pumps to ensure forced convection. Moreover, both the anode and cathode compartments are provided with a plastic mesh turbulence promoter. The flow is therefore assumed fully turbulent and a uniform velocity profile is assumed at the inlet. However, for simplification, these devices are not represented in the simulation domain. Although the turbulence promoter should actually influence the bubble population, no reference has been found on its effects.

Two additional simplifying assumptions result from the pilot facility operating conditions. First, a high recirculation rate is imposed in both compartments, leading to a low $\mathrm{SO}_{2}$ conversion rate per pass, typically $5 \%$, as given by the mass balance calculation described in [9]. The anolyte composition, $\mathrm{C}_{\mathrm{SO} 2}=0.24 \mathrm{~mol} / \mathrm{l}$, is therefore assumed to remain constant along the cell, in order to achieve nearly uniform current distribution along the electrode. Furthermore, the catholyte loop is provided with an efficient phase separation device. The gaseous $\mathrm{H}_{2}$ volume fraction can therefore be neglected at the cell inlet.

\subsection{Governing equations}

Current density, eqn. (1), is calculated by the finite-element code Flux-Expert ${ }^{\mathrm{TM}}$ solving the Laplace equation for potential, eqn. (2) throughout the computational domain. 


$$
\begin{gathered}
\vec{j}=-\sigma \vec{\nabla} \phi \\
\vec{\nabla} \cdot(-\sigma \vec{\nabla} \phi)=0
\end{gathered}
$$

where $\phi$ is the electrical potential in $\mathrm{V}$ and $\sigma$ the electrical conductivity in S.m ${ }^{-1}$.

The $\mathrm{SO}_{2}$ oxidation process is kinetically limited, inducing a non-negligible anodic overvoltage, given by a Tafel electrokinetic law [11]. In order to account for the secondary potential distribution prevailing in the domain, potential jumps are calculated at the anode/fluid interface thanks to the interfacial-type elements provided within the solver. These zero-width finite elements allow electrical potential discontinuities to be managed, as described in ref. [7]. The same formalism is used at the cathode/fluid interface as well to model the proton reduction overvoltage.

The fluid electrical conductivity $\sigma$ is assumed to be linearly dependent on temperature in all fluid zones and in the membrane as well. The linear law coefficients were fitted on impedance spectroscopy measurements performed over a wide range of temperatures and $\mathrm{H}_{2} \mathrm{SO}_{4}$ concentrations. The catholyte conductivity is also dependent on the gas volume fraction $\alpha_{2}$ following the Bruggeman relation:

$$
\sigma_{\left(T, \alpha_{2}\right)}=\sigma_{T, 0}\left(1-\alpha_{2}\right)^{1.5}
$$

The materials conductivity values à $50^{\circ} \mathrm{C}$ are given in Table 1 .

Table 1: $\quad$ Materials conductivity at $50^{\circ} \mathrm{C}$.

\begin{tabular}{|c|c|c|c|}
\hline & $\mathrm{CMX}$ & $30 \% \mathrm{wt} \mathrm{H} \mathrm{SO}_{4}$ & $50 \%$ wt $\mathrm{H}_{2} \mathrm{SO}_{4}$ \\
\hline$\sigma\left(\mathrm{S} . m^{-1}\right)$ & 17,5 & 98,5 & 78,5 \\
\hline
\end{tabular}

Both the temperature field and gas volume fraction distribution are computed by Fluent ${ }^{\mathrm{TM}}$ during the hydrodynamic iteration process.

The steady-state fluid mechanics problem is solved using the Fluent ${ }^{\mathrm{TM}}$ EulerEuler multiphase model in the fluid domains. Mass, momentum and energy balances, the general forms of which are given by eqn. (4), (5), and (6), are solved for both the liquid and the gas phases. In solid zones the energy equation reduces to the simple heat conduction problem with heat source. By convention, $i=1$ designates the $\mathrm{H}_{2} \mathrm{SO}_{4}$ continuous liquid phase whereas $\mathrm{H}_{2}$ bubbles constitute the dispersed phase $(i=2)$.

$$
\begin{gathered}
\vec{\nabla} \cdot\left(\alpha_{i} \rho_{i} \vec{u}_{i}\right)=S_{i} \\
\alpha_{i} \rho_{i}\left(\vec{u}_{i} \cdot \vec{\nabla}\right) \vec{u}_{i}=-\alpha_{i} \vec{\nabla} P+\vec{\nabla} \cdot \overline{\overline{\tau_{i}}}+\alpha_{i} \rho_{i} \vec{g}+\sum_{j} K_{j i}\left(\vec{u}_{j}-\vec{u}_{i}\right) \\
\vec{\nabla} \cdot\left(\rho_{i} C_{P_{i}} \alpha_{i} \vec{u}_{i} T_{i}\right)=\vec{\nabla} \cdot\left(\lambda_{i}^{e f f} \vec{\nabla} T_{i}\right)+\vec{\nabla} \cdot\left(\overline{\overline{\tau_{i}}} \cdot \vec{u}_{i}\right)+S_{Q_{i}}+\sum_{j} Q_{j i}
\end{gathered}
$$

The source term $S_{i}$ in eqn (4) is zero in the overall domain but in the $\mathrm{H}_{2}$ release zone. The source term $S_{Q i}$ in eqn (6) stands for the heat dissipation rate inherent 
in the electrokinetic processes. The last terms on the right-hand side of eqn. (5) and eqn. (6) represent the interphase interactions, where $\mathrm{Kji}$ is computed according to the Schiller and Naumann model for the drag coefficient, as in ref. [5], and Qji using the default FLUENT assumptions.

The renormalized $k$ - $\varepsilon$ model of FLUENT is used to compute the Reynolds stress tensors $\tau_{i}$ and the effective properties for each phase. A $k-\varepsilon$ turbulence model is also used in [3] and [8]. A less CPU-consuming model based on mixture-lengths is used in [4].

\subsection{Interphase and source terms}

In the Westinghouse electrochemical step, hydrogen is released at the cathode interface. In the numerical study, bubble generation is assumed to be localized in the first row of fluid cells neighbouring the electrode. In this special zone analogue to a boundary layer, the rate of gas production is assumed equal to the rate of the reduction process. It is modelled by the source term $S_{2}$ of the dispersed phase mass-balance equation, assuming a 100\% Faradic yield.

$$
S_{2}=\frac{M_{H_{2}}}{2 e F}(\vec{j} \cdot \vec{n})_{\text {int }}=-S_{1}
$$

where $M_{\mathrm{H} 2}$ is the gas molar mass $\left(210^{-3} \mathrm{~kg} \cdot \mathrm{mol}^{-1}\right), F=96485 \mathrm{C}^{-\mathrm{mol}^{-1}}$ is the Faraday constant and $e=10^{-4} \mathrm{~m}$ the width of the $\mathrm{H}_{2}$ release zone. The interfacial current density is calculated by Flux-Expert ${ }^{\mathrm{TM}}$.

The assumption $S_{1}=-S_{2}$ was first made by analogy with boiling, although no significant difference in the simulation results was observed by assuming $S_{l}=0$.

Also provided by the finite element solver is the source term $S_{Q}$ in the energy balance, eqn. (6). Except within the $\mathrm{H}_{2}$ release zone, the volumetric heat flux corresponds to heat losses by Joule effect in the conducting materials. In zone 2 , the heat dissipated by the irreversible interfacial processes is computed instead.

$$
S_{Q}=\eta \times(\vec{j} \cdot \vec{n})_{y=e}
$$

\subsection{Boundary conditions}

Material properties are assumed to be constant in the CFD simulation. Data representative of the operating conditions frequently described in the literature $[9,11]$ are used: the cell is operated under atmospheric pressure conditions with sulphuric acid solutions at $30 \mathrm{wt} \%$ in the cathode compartment and $50 \mathrm{wt} \%$ in the anode compartment, as recommended by the Westinghouse cycle efficiency evaluations. The desired specific flow-rate is imposed at the fluid inlet, where the temperature is maintained at $323 \mathrm{~K}$. Adiabatic conditions prevail at the domain boundaries.

A monodisperse population of spherical $\mathrm{H}_{2}$ bubbles is assumed. The bubble diameter was not measured and was arbitrarily set. The gas flow rate was computed from the current density, but regarding bubble impulsion we had a degree of freedom. Thus a constant departure angle $\theta$ is imposed to help compute the initial velocity components in the three directions of space (fig. 2). We 
assume therefore that bubbles are ejected perpendicularly ( $y$-direction) and that they are deviated from an angle $\theta$ in the flow direction by the convective flow. Since bubbles are not allowed to evolve along the surface (i.e. in the $x z$-plane), the $x$-component of the departure velocity is zero.

Regarding the electrokinetic problem, an imposed current supply is assumed at the anode corresponding to a uniform current density of 2000 A.m ${ }^{-2}$. The cathode potential is set at $0 \mathrm{~V}$.

CFD simulations were performed in single precision while the electrokinetic problem was solved in double precision. Double precision is required since current density is post-processed from the potential distribution and careful meshing is necessary as shown by Caire and Chifflet [12].

\section{Parameter study}

From a purely electrokinetic point of view (i.e. for single-phase flow), the calculated cell voltage equal to $0.732 \mathrm{~V}$ is mainly attributed to $\mathrm{SO}_{2}$ oxidation kinetics which is responsible for the anodic overvoltage (fig. 3).

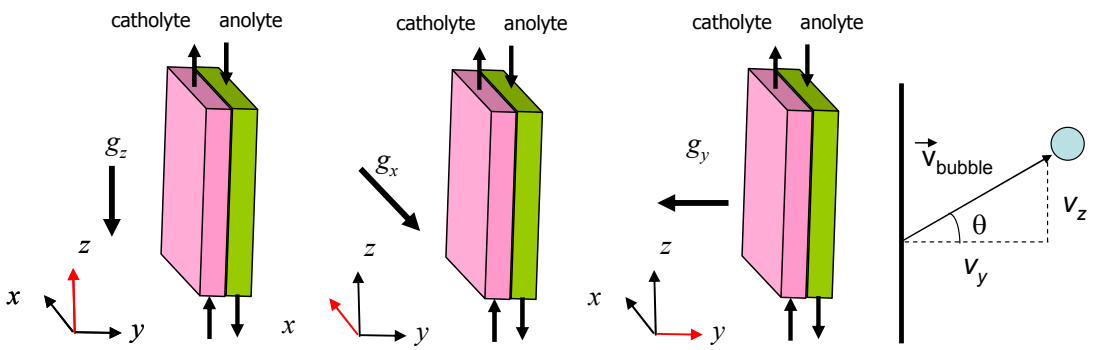

Figure 2: Schematic representation of the cell orientations (left) and bubble departure from the electrode surface (right).

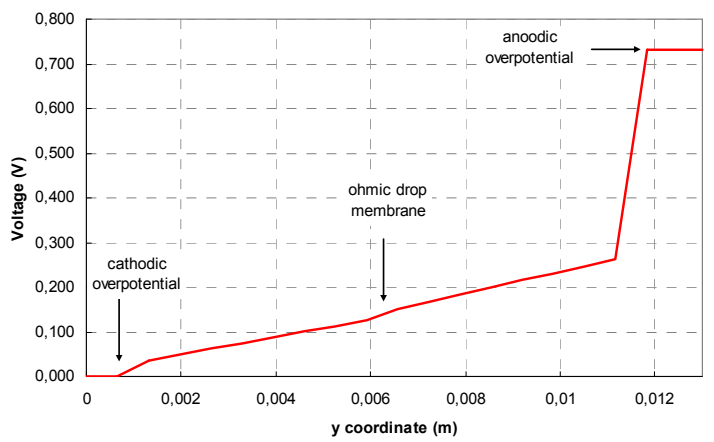

Figure 3: Cell potential distribution for single-phase flow $(x=0.02, z=$ $0.08)$. 
The model was used to study the process sensitivity to the major unknown parameters: the $\mathrm{H}_{2}$ bubble size $(50-200 \mu \mathrm{m})$ and their departure angle $\left(30-60^{\circ}\right)$. The effect of cell orientation and electrolyte flow rate in the range of the pilot facility operating conditions $\left(75-200 \mathrm{~L}^{-1} \mathrm{~h}^{-1}\right)$ was also investigated. Process sensitivity is measured in term of cell voltage and gas fraction maximum and average values. Gaseous phase spatial distribution is also examined. Major simulation results are summarized in table 2.

Three kinds of cell orientation were studied in reference with gravity (fig. 2): i) vertical gas-evolving electrode, like in prior work [3-5,7], ii) gravity vector oriented downward in $x$-direction, which is the configuration of the pilot facility, and iii) horizontal cathode facing upward. They are referred to as $g_{z}, g_{x}$ and $g_{y}$ respectively. For each case the gas fraction distribution in $y z$-plane within the cathode compartment is shown in fig. 4.

When the flow direction is parallel to gravity $\left(g_{z}\right)$, a gas curtain remains confined near the electrode. Bubbles progressively spread within the liquid (in the $y$-direction) with increasing $z$. The maximum penetration depth is closed to $20 \%$ of the gap width. Additional simulations indicate that increasing flow rate confines the bubbles at the neighbouring electrode, but decreases the overall gas amount since bubbles are conveyed by the liquid flow. The same effect is, to a lesser extent, achieved by increasing the departure angle (Table 2).

In the second case $\left(\mathrm{g}_{\mathrm{x}}\right)$, the plume is still present but a second bubble layer develops in the $y$-direction, expanding in the $x$-direction according to the gravity field. The gas fraction is therefore increasing, outside the bubble curtain, with increasing distance from the electrode.

Gas fraction and voltage distribution contours in the catholyte compartment are illustrated fig. 5. In the $g_{z}$ case (left), the gas fraction increases in the flow direction (z), whereas in the $g_{x}$ case (right) $\mathrm{H}_{2}$ is trapped at the top of the cell, under the effect of gravity. In this configuration, a slight asymmetry can be observed in the liquid flow direction. The voltage distribution reveals the corresponding local variations of the fluid conductivity.

In the case of the horizontal cathode facing upward $\left(g_{y}\right)$, the plume along the flow direction vanishes and gas accumulates just below the rigid membrane, where the simulation predicts a phase segregation $\left(\alpha_{2} \rightarrow 1\right)$. Convergence
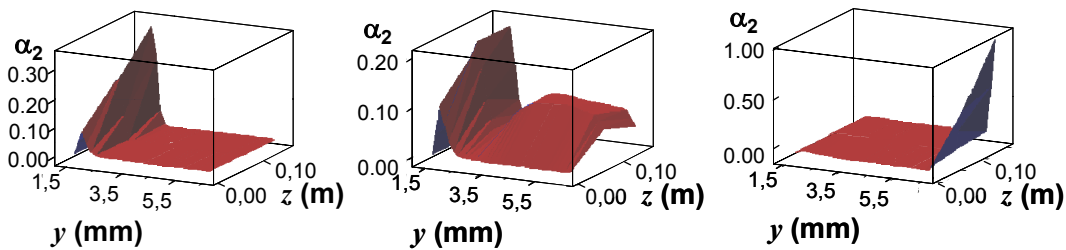

Figure 4: Gas distribution in the cathode compartment for $\mathrm{g}_{z}$ at $x=0.02$ (left), $\mathrm{g}_{\mathrm{x}}$ at $x=0.03$ (middle) and $\mathrm{g}_{\mathrm{y}}$ at $x=0.01$ (right) for $\theta=30^{\circ}$, $\mathrm{d}_{\text {bubble }}=100 \mu \mathrm{m}$, flow rate $75 \mathrm{~L} \cdot \mathrm{h}^{-1}$. 

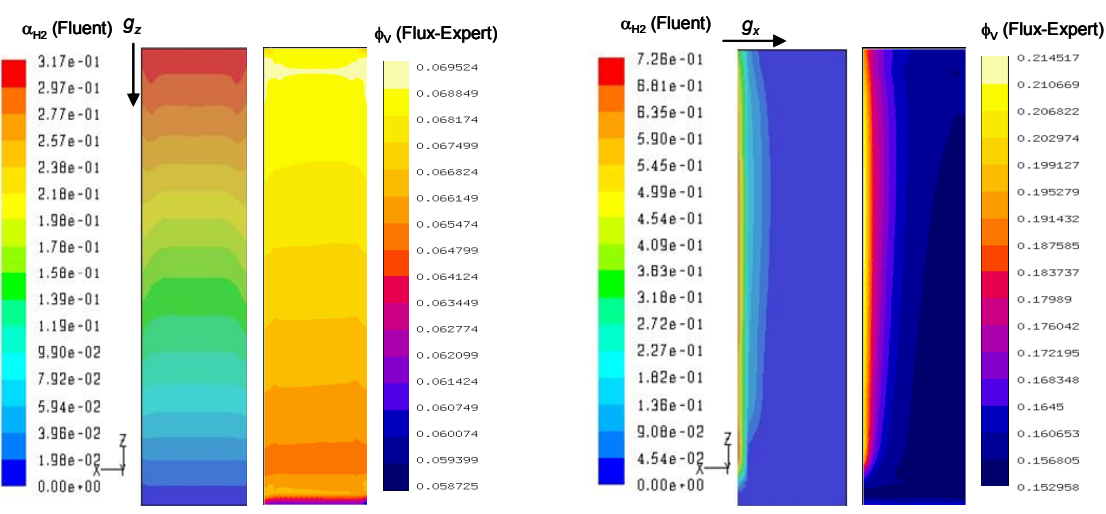

Figure 5: Surface plots of gas volume fraction $\alpha_{2}$ and voltage $\phi$ in the cathodic compartment for $\mathrm{d}_{\text {bubbles }}=100 \mu \mathrm{m}, \theta 30^{\circ}$, flow-rate $75 \mathrm{~L}$. $\mathrm{h}^{-1}$ in the $g_{z}$ (near the cathode) and $g_{x}$ (near the membrane) configurations.
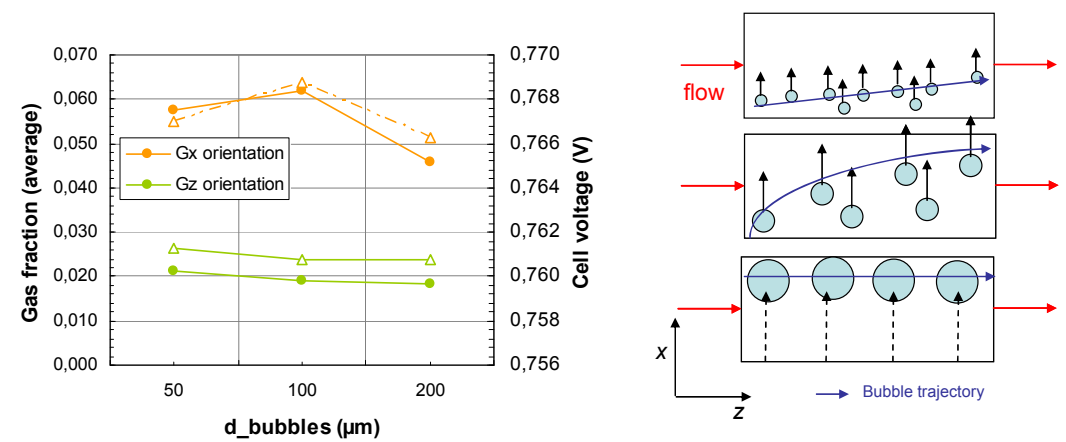

Figure 6: Left: gas holdup (circles) and cell voltage (triangles) evolutions with bubble size $\left(\theta 30^{\circ}\right.$, flow-rate $\left.75 \mathrm{~L} \cdot \mathrm{h}^{-1}\right)$ in the $g_{x}$ (top) or $g_{z}$ (bottom) orientation. Right: illustration of the bubbles trajectories in the $g_{x}$ configuration for small (top), medium (middle) and large (bottom) bubbles.

troubles were encountered during the resolution process since the dispersed phase became predominant. The same difficulties were encountered for the same reason when simulating the $g_{x}-\mathrm{d}_{\text {bubble }}=200 \mu \mathrm{m}$ configuration.

The variation of the average gas fraction and the cell voltage with the bubble size is depicted in the left part of fig. 6 for the $g_{z}$ and $g_{x}$ configurations.

In the $g_{z}$ configuration, the gas holdup (and correlatively the cell voltage) decreases with the bubble diameter, according to the bubbles rising velocity evolution. 
In the $g_{x}$ configuration, the bubble free rising direction is perpendicular to the flow direction. Increasing the bubble size is first leading to an increase of the gas holdup. Indeed, the bigger bubbles trajectories are more deviating from the flow trajectory, thus increasing their residence time in the cathode compartment (fig. 6 , right). If the bubble diameter is further increased, the reverse phenomenon is observed. In this case, bubbles are rapidly rising from the cathode to the top of the, finite-width, electrolyser, from which they are conveyed straightforwardly toward the cell outlet by the convective flow, with a reduced residence time.

Table 2: $\quad$ Parameter study assumptions and results.

\begin{tabular}{|c|c|c|c|c|c|c|}
\cline { 2 - 7 } \multicolumn{1}{c|}{} & $\mathbf{d}_{\text {bubbles }}$ & Flow rate & $\boldsymbol{\theta}$ & $\boldsymbol{\alpha}_{2, \text { max }}$ & $\boldsymbol{\alpha}_{2, \text { mean }}$ & $\Delta \boldsymbol{\phi}$ \\
\hline monophasic & $/$ & $75 \mathrm{~L} \cdot \mathrm{h}^{-1}$ & $/ 0^{\circ}$ & $/$ & $/$ & $0.732 \mathrm{~V}$ \\
\hline $\boldsymbol{g}_{\boldsymbol{x}}$ & $50 \mu \mathrm{m}$ & $75 \mathrm{~L} \cdot \mathrm{h}^{-1}$ & $30^{\circ}$ & 0.458 & 0.057 & $0.767 \mathrm{~V}$ \\
\hline $\boldsymbol{g}_{\boldsymbol{x}}$ & $100 \mu \mathrm{m}$ & $75 \mathrm{~L} \cdot \mathrm{h}^{-1}$ & $30^{\circ}$ & 0.726 & 0.062 & $0.769 \mathrm{~V}$ \\
\hline $\boldsymbol{g}_{\boldsymbol{x}}$ & $200 \mu \mathrm{m}$ & $75 \mathrm{~L} \cdot \mathrm{h}^{-1}$ & $30^{\circ}$ & 0.936 & 0.046 & $0.766 \mathrm{~V}$ \\
\hline $\boldsymbol{g}_{\boldsymbol{x}}$ & $100 \mu \mathrm{m}$ & $75 \mathrm{~L} \cdot \mathrm{h}^{-1}$ & $60^{\circ}$ & 0.774 & 0.064 & $0.768 \mathrm{~V}$ \\
\hline $\boldsymbol{g}_{\boldsymbol{x}}$ & $100 \mu \mathrm{m}$ & $200 \mathrm{~L} \cdot \mathrm{h}^{-1}$ & $30^{\circ}$ & 0.258 & 0.018 & $0.757 \mathrm{~V}$ \\
\hline$g_{y}$ & $100 \mu \mathrm{m}$ & $75 \mathrm{~L} \cdot h^{-1}$ & $30^{\circ}$ & 0.996 & 0.044 & $0.773 \mathrm{~V}$ \\
\hline $\boldsymbol{g}_{z}$ & $50 \mu \mathrm{m}$ & $75 \mathrm{~L} \cdot \mathrm{h}^{-1}$ & $30^{\circ}$ & 0.386 & 0.021 & $0.761 \mathrm{~V}$ \\
\hline $\boldsymbol{g}_{z}$ & $100 \mu \mathrm{m}$ & $75 \mathrm{~L} \cdot \mathrm{h}^{-1}$ & $30^{\circ}$ & 0.367 & 0.019 & $0.761 \mathrm{~V}$ \\
\hline $\boldsymbol{g}_{z}$ & $200 \mu \mathrm{m}$ & $75 \mathrm{~L} \cdot \mathrm{h}^{-1}$ & $30^{\circ}$ & 0.350 & 0.018 & $0.761 \mathrm{~V}$ \\
\hline $\boldsymbol{g}_{z}$ & $100 \mu \mathrm{m}$ & $75 \mathrm{~L} \cdot \mathrm{h}^{-1}$ & $60^{\circ}$ & 0.357 & 0.015 & $0.760 \mathrm{~V}$ \\
\hline $\boldsymbol{g}_{z}$ & $100 \mu \mathrm{m}$ & $200 \mathrm{~L} \cdot \mathrm{h}^{-1}$ & $30^{\circ}$ & 0.295 & 0.014 & $0.757 \mathrm{~V}$ \\
\hline
\end{tabular}

\section{Conclusion}

A parameter study of the two-phase filter-press electrolyser was carried out in the scope of optimizing the Westinghouse electrochemical step. The process sensitivity to biphasic phenomena was qualitatively assessed using a multiphysics model.

As compared with the "monophasic" case (i.e. the purely electrokinetic problem solved by Flux-Expert, without CFD coupling) and in the range of conditions explored, hydrogen bubbles are responsible for an additional overvoltage ranging from 25 to $40 \mathrm{mV}$. Among the investigated parameters, the electrolyte flow rate appeared to have the greatest influence on the cell voltage, irrespective of the cell orientation.

The model has demonstrated its versatility, allowing a detailed description of the various phenomena involved, but failed in predicting nearly segregated configurations for which numerical difficulties occurred. As the gas fraction approaches 1 , the electrolyte conductivity tends towards 0 . Moreover, from a fluid mechanics standpoint, the validity of the Euler-Euler model is questionable and a Volume of Fluid approach should be preferred.

Available experimental data, measured under fixed cell voltage conditions, exhibit unexpectedly low current densities. Indeed, although an ideal impermeable membrane was considered for the simulation, non-negligible $\mathrm{SO}_{2}$ crossover occurred during the experiments, due to the poor selectivity of the available membrane. Therefore, an appreciable fraction of the expected current 
density was consumed by side reactions induced by the sulphur species. The occurring of the latter side reactions, not taken into account in the model, prevents us from validating the two-phase model assumptions, unless a more efficient separator is used.

A detailed description of species transport, membrane exchanges and electrochemical processes is required as well to reach a sufficient degree of understanding of the electrolytic cell functioning, and therefore to complete the design and optimization of an industrial-scale process. Such a phenomenological model is currently being developed. Experiments have also been designed to improve the $\mathrm{SO}_{2}$ oxidation overvoltage model, which will be described in a future paper.

A phenomenological description of $\mathrm{H}_{2}$ bubbles evolving at industrial electrodes is currently in progress within the scope of the AMELHYFLAM project, and the qualitative phenomena highlighted by this simulation study will be helpful in designing the future cell instrumentation.

\section{Acknowledgements}

The authors are grateful to the ANR project AMELHYFLAM for financial support and to the LECNA team of the CEA Saclay laboratory for the supplied electrochemical properties.

\section{References}

[1] Trinidad P, Ponce de León C. \& Walsh F.C., The application of flow dispersion models to FM01-LC laboratory filter-press reactor. Electrochemica Acta, 52, pp. 604-613, 2006.

[2] Duerr M., Gair S., Cruden A. \& McDonald J., Dynamic electrochemical model of an alkaline fuel cell stack. Proc. of $16^{\text {th }}$ World Hydrogen Energy Conference, June 13-16, Lyon (France), 2006.

[3] Mandin P., Hamburger J., Bessou S. \& Picard G., Modelling and calculation of the current density distribution evolution at vertical gasevolving electrodes. Electrochemica Acta, 51, pp.1140-1156, 2005.

[4] Agranat V., Zhubrin S., Maria A., Hinatsu J., Stemp M. \& Kawaji M., CFD modelling of gas-liquid flow and heat transfer in a high pressure water electrolyser system. Proc. of FEDSM, July 17-20, Miami (Florida) 2006.

[5] Mat M., Aldas K. \& Ilegbusi O., A two-phase model for hydrogen evolution in an electrochemical cell. Int. Jal of H2 Energy, 29, pp. 1015 1023, 2004.

[6] Roustan H., Caire J.P., Nicolas F. \& Pham P., Modelling coupled transfers in an industrial fluorine electrolyser. Jal of Applied Electrochemistry, 28, pp. 237-243, 1998.

[7] Jomard J, Feraud J.P., Morandini J., Du Terrail Couvat Y. \& Caire J.P., Hydrogen filter press electrolyser modelled by coupling Fluent and Flux Expert codes. Jal of Applied Electrochemistry, 38, pp. 297-308, 2008. 
[8] Espinasse G., Peyrard M., Nicolas F. \& Caire J.P., Effect of hydrodynamics on Faradaic current efficiency in a fluorine electrolyser, Jal of Applied Electrochemistry, 37, pp. 77-85, 2007.

[9] Rivalier P., Charton S., Ode D., Duhamet J., Boisset L., Pabion J.L., Gandi F. \& Croze J.P., Design study of a pilot test plant for hydrogen production by a hybrid thermochemical process, Proc. of the16th Int. Conf. on Nuclear Engineering, May 11-15, Orlando (Florida), 2008.

[10] Brown C.J., Pletcher D., Walsh F.C., Hammond J.K. \& Robinson D., Local mass transport effects in the FM01 laboratory electrolyser. Jal of Applied Electrochemistry, 22, pp. 613-619, 1992.

[11] Appleby A.J. \& Pinchon B., The mechanisms of the electrochemical oxidation of sulphur dioxide in sulphuric acid solutions. Jal of Electroanalytical Chemistry, 95(1), pp. 59-71, 1979.

[12] Caire J.P. \& Chifflet H., Meshing noise effect in design of experiments using computer experiments, Environmetrics, 13(1), pp. 1-8, 2002. 